\title{
Healthcare Scheduling by Data Mining: Literature Review and Future Directions
}

\author{
Maria M. Rinder ${ }^{1 *}$, Gary Weckman ${ }^{1}$, Diana Schwerha', Andy Snow ${ }^{2}$, \\ Peter A. Dreher ${ }^{4}$, Namkyu Park ${ }^{1}$, Helmut Paschold ${ }^{3}$ and William Young ${ }^{1}$ \\ ${ }^{1}$ Industrial and Systems Engineering, Russ College of Engineering and Technology, \\ ${ }^{2} J$. Warren McClure School of Information and Telecommunication Systems, \\ ${ }^{3}$ Department of Social and Public Health, College of Health Sciences and \\ Professions, Ohio University, Athens, Ohio 45701 \\ ${ }^{4}$ College of Science and Engineering, Central State University, Wilberforce, \\ Ohio 45384, USA
}

Submitted August 2011. Accepted for publication April 2012.

\begin{abstract}
This article presents a systematic literature review of the application of industrial engineering methods in healthcare scheduling, with a focus on the role of patient behavior in scheduling. Nine articles that used mathematical programming, data mining, genetic algorithms, and local searches for optimum schedules were obtained from an extensive search of literature. These methods are new approaches to solve the problems in healthcare scheduling. Some are adapted from areas such as manufacturing and transportation. Key findings from these studies include reduced time for scheduling, capability of solving more complex problems, and incorporation of more variables and constraints simultaneously than traditional scheduling methods. However, none of these methods modeled no-show and walk-ins patient behavior. Future research should include more variables related to patient and/or environment.
\end{abstract}

Keywords: heuristic methods, healthcare scheduling, patient behaviour

\section{INTRODUCTION}

\subsection{Background}

A healthcare provider operates in a regulated industry in which the quality of its performance is evaluated in terms of the services rendered to the patient and the effectiveness of the provider's process. A measure of process effectiveness is the

*Corresponding author: Maria M. Rinder, Industrial and Systems Engineering, Russ College of Engineering and Technology, Ohio University, Athens, OH 45701. Phone: (740)593-1539. Fax: (740)593-0659. E-mail: magdarinder@hotmail.com. Other authors: weckmang@ohio.edu, schwerha@ohio.edu, snowa@ohio.edu 
number of patients that visit the practice per day. Patient satisfaction is an important measure of quality of healthcare. A well designed scheduling system could increase patient satisfaction, access to care, as well as the effectiveness of healthcare operations [6, 7 and 8]. Surveys suggest that excessive waiting time is a major reason for patient's dissatisfaction [9]. In addition to clinical competence, a reasonable waiting time is expected [10]. Patient satisfaction is influenced by the performance of the scheduling system. The scheduling process is a critical element for the patients and practitioners, and often, is the first contact between the patient and provider [11]. Patients want to be seen on time by physicians who in turn want a system to triage patients efficiently [11].

Patients who schedule appointments face direct and indirect waiting times [3]. Indirect waiting time is the time between a patient's request and the actual appointment time. Long indirect waiting times prevent patients with acute needs from being seen by the provider in a timely manner [12]. The direct waiting time comes between the scheduled appointment time and the time the patient receives care. The scheduling system can affect both direct and indirect waiting times. Whereas direct waiting is an inconvenience to the patient, excessive indirect wait can pose a serious safety concern [13]. If patients cannot receive healthcare service at the time needed, their condition can deteriorate, perhaps even become life-threatening.

In developing patient schedules, there are a number of external factors that a provider cannot control, such as no-show appointments and cancellations, emergency appointments, and walk-ins. These factors are determined by patients' needs. No-shows and walk-ins disrupt resource utilization plans and hence operational efficiency.

\subsection{Industrial Engineering Methods Applied to Healthcare Scheduling}

This paper discusses the application of industrial engineering techniques to improve scheduling in healthcare. We define two categories of healthcare scheduling: work (patient) scheduling and provider resource scheduling. In health systems, the patient is the work. The work/patient can be scheduled for those departments where there are slots available (e.g., outpatient clinic). For other departments, the work scheduling is simply based on first-in first-out or triage assessments (e.g., emergency room). Work scheduling has to be accompanied by resource scheduling which includes staff, equipment, and room scheduling. This schedule is based on the conditions for each department, such as number of slots available, time required for each slot, equitability between personnel (number of hours to be worked, days in a week, and holidays).

\subsubsection{Work Scheduling}

Patient appointments are scheduled based on the number of appointments slots available. The number of appointments available is established based on the type of work, such as regular visits, follow-up visits, tests and procedures, education sessions, and the number of providers available by hour and day of the week. It is the current practice that new appointments are made over the phone or internet and the patient has to be fit into a pre-existing schedule [6]. Follow-up appointments can be made at the end of the visit or by phone/internet. Appointments are made until all available slots are filled up. Scheduling can be performed using a manual system (e.g., appointment book) 
or a computerized system. Both methods have to search for an available spot based on patient requirements. The manual system requires that the scheduler have specific knowledge and job experience. The computerized system can do the same albeit electronically.

\subsubsection{Staff Scheduling}

Staff scheduling can be performed only after the staff requirements are determined. Staff scheduling assigns each staff member to a pattern of work and leave days [14]. We define two types of staff scheduling: cyclical and non-cyclical. Cyclical scheduling assigns the same pattern over a certain number of days or weeks, with an advantage of employee's familiarity with the schedule, and a disadvantage of lack of flexibility in accommodating the demand and staff needs. Non-cyclical scheduling generates new schedules over a short period of time (e.g., two weeks) based on the demand and available staff. Although it can accommodate change in demand and staff needs, noncyclical scheduling requires more planning time than cyclical scheduling. Development of cyclical and non-cyclical schedules has used heuristics, trial-and-error, or optimization techniques [14].

\subsection{An Overview of Healthcare Scheduling Method Literature}

In this section, we review the proposed methods used in the healthcare scheduling literature. The early articles $[15,16]$ are based on mathematical programming methods and queuing theory. These studies assume that the conditions in which work is performed were static. Lindley (1952) used the case of a single server and random patient arrivals, and concluded that scheduling at regular intervals improves system performance [15].

Following Lindley's model, more mathematical models were developed in which dynamic conditions were added to simulate more closely the actual environment. The mathematical model proposed by Jansson [17] featured an individual block with constant appointment times. Soriano [18] later modelled multiple-block/fixed intervals. Subsequently, Mercer [19] modelled individual-block and late arrivals, also considering the probability of patient no-show. Later mathematical models use dynamic programming, variable-block/fixed interval [20], multi-server queuing models with nonhomogeneous arrivals [21], a queuing system with multiple doctors and random arrival time [22], and two-stage stochastic linear programming to determine the optimal appointment intervals [23].

Bailey [16] used a simplified queuing technique assuming fairly static conditions such as appointment times at regular intervals based on the average consultation time, patients arriving punctually for their appointments, patients being seen in the order they arrive, and the provider seeing only one patient at the time. Some variables such as number of patients visiting the clinic, length of appointment interval, and number of patients waiting at any time, were varied for comparison reasons. The goal of the model was to reduce patient waiting and consultant idle time. Bailey's model used a Monte Carlo simulation technique and is considered one of the first simulation models. It was further developed to include the scheduling of groups of patients based on appointment length, and break time for consultants [24]. 
Based on a mathematical model, Welch [25] concluded that the factors that may influence appointment scheduling are punctuality of medical staff and patients, and appointment intervals (time between appointments).

More simulation models were developed a few years after Bailey's model. Fetter and Thompson [26] added some of the variables that were considered fixed in the previous models, such as patient punctuality, number of appointments, no-show rates, walk-in rates, appointment intervals, and patient loads. Vissers and Wijngaard [27] modelled a system based on five variables, mean consultation time, patient's punctuality, number of appointments, and "system earliness" that is allowing patients to arrive earlier than the expected moment of treatment. Ho and Lau [28, 29, and 30] developed some of the most comprehensive models by including 50 appointment rules. They concluded that no-show, service time, and number of patients are the major factors affecting the system performance.

Klassen and Rohleder [31, 32] simulated different variance patients (patients with different service times) and fixed appointment intervals. They concluded that "lowvariance" patients (patients with low variance in service time) assigned at the beginning of the appointment session may perform better. They also included two slots for emergency, and scheduler error when classifying patients.

These techniques showed improved scheduling results, however, with limitations particularly in lack of generality. The studies analyzed a specific clinic, accounting for specific environmental conditions that may not apply to other clinics. A second limitation is the use of overly simplistic models, such as single server, single-phase, and nonrandomization of arrival patterns. A third limitation is that none of these models accounted for the effects of walk-ins, no-shows, or emergencies which are common in real life.

This review paper focuses on analysis of more complex methods used in healthcare scheduling that can improve scheduling results through the inclusion of greater variability and complexity.

\section{METHODS}

\subsection{Research Question}

The specific research question of this literature study is: Does any of these techniques analyze data that can be used to improve scheduling in healthcare?

\subsection{Search Strategy}

To retrieve relevant studies from peer-reviewed literature, Pub Med electronic database was searched. The search included articles published up to August 2011 using the following keywords: MODEL AND (DATA) AND (HEALTHCARE OR MEDICAL) and (SCHEDULING). In addition to the electronic search, bibliographies in the relevant papers were reviewed to identify additional studies.

\subsection{Selection Criteria}

The studies that were included in our review met all of the following requirements: (a) the paper described application of data modeling technique to improve scheduling in healthcare, and the modeling technique had to be a data mining technique; (b) the modeling technique was applied to a real database; and (c) the paper was a full report published in 
English in a peer-reviewed journal. After article titles were identified from the database, the abstracts were reviewed for possible inclusion. For the abstracts selected for inclusion, or abstracts that provided insufficient information, full papers were retrieved. The same procedures were applied to the articles that were selected from bibliographies. After reading the full article, the articles that met our criteria were selected.

\subsection{Data Extraction}

The following items were documented from the included studies: locations (U.S. versus Non-U.S.), study designs, study periods, number of exposed groups, exposure variables, and outcomes.

\subsection{Quality Assessment}

The Epidemiological Appraisal Instrument (EAI) developed by Genaidy (2004) was used to critically appraise the methodological quality of the studies. The EAI [33] consists of 43 questions grouped into five scales:

1. Reporting (17 items),

2. Subject selection (7 items),

3. Measurement quality (10 items),

4. Data analysis (7 items), and

5. Generalization of results (2 items).

Every item in the EAI was rated for a given study using one of the following options:

1. Not applicable (not included in score calculation),

2. Yes (2 points),

3. Partial (1 point),

4. No (0 points), and

5. Unable to determine ( 0 points).

The final score for each scale was taken as the average of the values recorded for each item.

\section{RESULTS}

\subsection{Identification of Studies}

The selection criteria enumerated in Section 2.3 was applied throughout the identification process. Following the search of the electronic database, a total of 2,174 citations were obtained, of which 2,066 citations were excluded for irrelevance to the topic of the review. The remaining 108 abstracts were reviewed, of which 73 were further excluded for not meeting the selection criteria. Among the 35 full papers retrieved, 26 were excluded for not meeting the selection criteria. Nine relevant studies were finally identified. Figure 1 shows a flow diagram of studies accepted and rejected during this identification phase. 


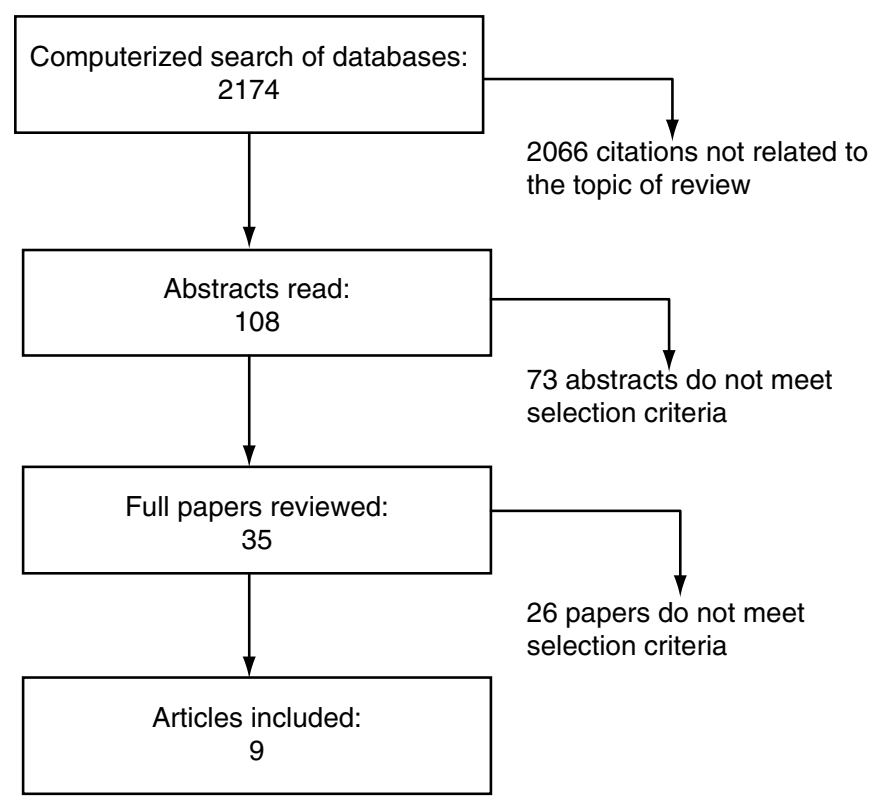

Figure 1. Flow diagram of articles selected in the process of study identification.

We arranged the nine articles in two groups. The first group consisted of six studies that describe techniques for patient scheduling [34, 35, 36, 37, 38 and 39]. The second group consisted of three studies that describe techniques for nurse scheduling [40, 41 and 42].

\subsection{Description of Evidence}

Description of evidence was summarized for all nine studies in terms of intervention, outcomes, study design, and main results. The description of evidence for Group 1, the six studies of patient scheduling, is listed in Table 1. These studies have outcomes of measured patient waiting time, idle time for staff or devices, and overall duration. In particular:

1. Whereas Chien et al. [37] applied a genetic algorithm to scheduling, Podgorelec and Kokol [34] introduced a genetic algorithm and machine learning approach to solve the scheduling problem.

2. Two of the studies $[38,39]$ introduced mathematical models to schedule patients and personnel in hospital services.

3. Kaandorp and Koole [36] used a local search procedure to find the optimal schedule with a weighted average of expected waiting times for patients, idle time for doctors, and tardiness as objectives.

4. A data mining approach to support simulation modeling of patient flow was introduced by Isken and Rajagopalan [35]. 
Table 1. Description of evidence for patient scheduling (Group 1)

\begin{tabular}{|c|c|}
\hline Source & Description \\
\hline \multicolumn{2}{|c|}{ Chakraborty et al. 2010 [39] } \\
\hline Intervention & $\begin{array}{l}\text { Use of dynamic programming for scheduling including patient } \\
\text { no-shows and general service time distributions. }\end{array}$ \\
\hline Outcome & $\begin{array}{l}\text { Maximize the expected revenue for patients seen minus costs } \\
\text { for patient waiting and staff overtime. }\end{array}$ \\
\hline Study design & Intervention \\
\hline Study population & Hospital patients \\
\hline Methods & $\begin{array}{l}\text { - Includes arbitrary service time distributions. } \\
\text { The objective function is unimodal for general service } \\
\text { time and the unimodality is independent of the type of } \\
\text { the service time distribution. } \\
\text { Develops a special case of gamma service times which } \\
\text { requires significanty less computation. }\end{array}$ \\
\hline Main results & $\begin{array}{l}\text { - It shows how the computational needs can be reduced } \\
\text { significantly when service times are approximated by a } \\
\text { gamma distribution. } \\
\text { The value of the maximum profit decreases with } \\
\text { increasing variance of the service time. }\end{array}$ \\
\hline
\end{tabular}

Chien et al., 2008 [37]

Intervention

Outcome

Study design

Study population

Methods
- Use of a genetic algorithm(GA) to solve a hybrid shop scheduling problem to increase service quality.

- $\quad$ Mixed integer programming model for validation purposes.

Reduce patient waiting time and improve operation efficiency. Intervention

Patients in a physical therapy facility

- $\quad$ Patients are considered jobs and medical resources machines.

- Considered medical resources as identical and set in parallel.

- GA is employed for sequencing module to establish new search points in historical information based on following steps:

$\circ$ A set of initial solutions is encoded as a set of chromosomes called population.

$\circ$ Crossover and mutation are applied to the population to generate offspring from parents. 
484 Healthcare Scheduling by Data Mining: Literature Review and Future Directions

Table 1. Description of evidence for patient scheduling (Group 1) (Contined)

\begin{tabular}{|c|c|}
\hline Source & Description \\
\hline \multicolumn{2}{|c|}{ Chien et al., 2008 [37] } \\
\hline Methods & $\begin{array}{l}\text { An evaluation and scaling function is used to assign a } \\
\text { fitness value to each chromosome. } \\
f(k)=w_{w} * W_{\max }(k)+w_{c} * C_{\max }(k)+P(k) \\
\text { where } W_{\max }(k) \text { is the maximum waiting time for } \\
\text { chromosome } \mathrm{K} \text {, } \\
C_{\max }(k) \text { is the makespan of chromosome } k \text {, } \\
\mathrm{P}(\mathrm{k}) \text { is a penalty function of chromosome } k \text {. } \\
w_{w} \text { is the weight of maximum waiting time, and } \\
w_{c} \text { is the weight of makespan. } \\
\text { Selection mechanism is employed to improve the solu- } \\
\text { tion quality. } \\
\text { The roulette wheel approach is used for population } \\
\text { selection. } \\
\text { To validate the solution, a mixed integer program- } \\
\text { ming is used to provide the optimal solution. } \\
\text { Objectives: to minimize maximum waiting time } \\
\text { and minimize the makespan. } \\
\text { Minimize } w_{w} * W_{\max }+w_{c} * C_{\max }\end{array}$ \\
\hline Main results & $\begin{array}{l}\text { - Both methods reveal identical waiting time in } \\
\text { both problems. } \\
\text { Average computation time for mixed integer pro- } \\
\text { gramming }(\mathrm{MIP}) \text { is: } \\
\quad 1.405 \mathrm{~s}(\mathrm{StDev}=1.916 \mathrm{~s}) \text { for } 10 \text { patients prob- } \\
\text { lem } \\
\quad 4496.505 \mathrm{~s}(\mathrm{StDev}=889.102 \mathrm{~s}) \text { for } 20 \text { patients } \\
\text { problem } \\
\text { - Average computation time for GA is less than } 10 \mathrm{~s} \\
\text { in both problems. }\end{array}$ \\
\hline
\end{tabular}

Ogulata et al., 2008 [38]

Intervention

Outcome

Study design

Study population
Development and testing of a hierarchical mathematical model for patient and staff scheduling

Maximize number of patients seen in a day, decrease waiting time for patients, and achieve fair distribution of patients among physiotherapist.

Intervention

90 patients who applied for treatment between January 15-22, 2007. 
Table 1. Description of evidence for patient scheduling (Group 1) (Contined)

\begin{tabular}{|c|c|}
\hline Source & Description \\
\hline \multicolumn{2}{|c|}{ Ogulata et al., 2008 [38] } \\
\hline Methods & $\begin{array}{l}\text { - Four therapists are available. } \\
\text { The problem was broken into three stages: } \\
1^{\text {st }} \text { stage: weekly patient selection is based on priori- } \\
\text { ty (high, medium, and low) and availability of phys- } \\
\text { iotherapist. } \\
2^{\text {nd }} \text { stage: assignment to physiotherapist. } \\
3^{\text {rd }} \text { stage: patient scheduling; each patient is } \\
\text { assigned to one time interval in a working day. } \\
\text { Using stage } 1 \text { model, } 54 \text { patients were selected based on } \\
\text { their priority. From these, } 44 \% \text { have high priority, } 49 \% \\
\text { medium, and } 7 \% \text { low. } \\
\text { In stage two, the patients are assigned to a physiothera- } \\
\text { pist as follows: } 14 \text { patients to } 1^{\text {st }} \text { therapist, } 14 \text { to } 2^{\text {nd }}, 13 \\
\text { to } 3^{\text {rd }} \text {, and } 13 \text { to the last one. }\end{array}$ \\
\hline Main results & $\begin{array}{l}\text { - The proposed algorithm scheduled } 6 \text { more patients than } \\
\text { the scheduling method used in the hospital, and sched- } \\
\text { uled } 24 \text { patients with high priority compared with } \\
15 \text { scheduled with the existing method. } \\
\text { - The proposed algorithm decreased patient waiting time. } \\
\text { - Limitations: the algorithm does not take into account } \\
\text { patient preference for day of the week. }\end{array}$ \\
\hline
\end{tabular}

Kaandorp and Koole, 2007 [36]

Intervention

Outcomes

Study design

Study population

Methods
A local search procedure in patient scheduling.

- Weighted average of expected waiting times of patients;

- Idle time of the doctor;

- Tardiness (lateness).

N/A

N/A

The formulas to calculate mean waiting time $W(x)$, idle time $I(x)$, and tardiness $L(x)$ are developed. The objective function becomes:

$$
\operatorname{MIN} \alpha_{W} W(x)+\alpha_{I} I(x)+\alpha_{L} L(x)
$$

where $\alpha_{W}, \alpha_{I}$ and $\alpha_{L}$ are the weights for mean waiting time, idle time, and tardiness.

- Since the number of solution is too big, a neighborhood is defined. 
Table 1. Description of evidence for patient scheduling (Group 1) (Contined)

\begin{tabular}{|c|c|}
\hline Source & Description \\
\hline \multicolumn{2}{|c|}{ Kaandorp and Koole, 2007 [36] } \\
\hline Main results & $\begin{array}{l}\text { A local search is started to find the most feasible solu- } \\
\text { tion in the neighborhood. A local minimum is found. } \\
\text { - Prove that the local minimum is the global minimum. } \\
\text { - Include an equal no-show probability for each patient. } \\
\text { The results of the proposed model give a better solution } \\
\text { than the two existing schedules. } \\
\text { - The variation in no-show probability influences the } \\
\text { scheduling. For a higher probability, the mean waiting } \\
\text { time, idle time and tardiness become larger. }\end{array}$ \\
\hline
\end{tabular}

Isken and Rajagopalan, 2002 [35]

Intervention

Outcome

Study design

Study population

Methods

Main results
Use of data-mining technique $-K$-means cluster analysis - to support a process of building computer simulation models. Effectiveness of data preprocessing and clustering methods. $\mathrm{N} / \mathrm{A}$

8261 patient visit records from several months in 1996.

- A master data table was created. It involves selection of patient based on the importance of certain variables and cases.

- The clustering problem was defined as following: to partition pattern vectors into a number of clusters such that each of them has at least one cluster member, and they are pair by pair disjoint and are mutually exhaustive. Euclidean distance function was used for two vectors.

- The most important variable is Diagnosis Related Groups (DRG).

- K-means algorithm is used to find $k$ optimal clusters in the data set. The function used is:

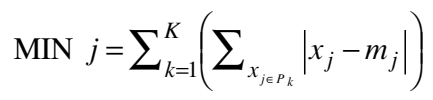

where $x_{j}$ is a dimensional pattern vector.

- For cluster selection, a measure named cluster purity was used. It uses the number of distinct DRGs appearing in a cluster.

- Data preparation affects positively the quality of solutions.

- The K-means algorithm completed the task in 3-5s (when implemented in ClustanGraphics 5) 
Table 1. Description of evidence for patient scheduling (Group 1) (Contined)

\begin{tabular}{|c|c|}
\hline Source & Description \\
\hline \multicolumn{2}{|c|}{ Podgorelec and Kokol, 1997 [34] } \\
\hline Intervention & $\begin{array}{l}\text { Scheduling algorithm based on genetic algorithm and } \\
\text { machine learning. }\end{array}$ \\
\hline Outcome & $\begin{array}{l}\text { Optimal schedule that can result in finishing all the activities } \\
\text { scheduled as soon as possible, with the least patient waiting } \\
\text { time and maximum device utilization (lowest overall duration). }\end{array}$ \\
\hline Study design & N/A \\
\hline Study population & 22 patients and 5 therapeutic devices were used. \\
\hline Methods & $\begin{array}{l}\text { - For every given problem, random test problems were } \\
\text { generated and executed. } \\
\text { - A number of random schedules were generated. } \\
\text { Each individual was evaluated for the fitness score. The } \\
\text { individuals with the best score were selected. } \\
\text { - The crossover procedure was applied until new individ- } \\
\text { uals fulfill the complete population. Each time when an } \\
\text { individual is added, another individual (with the lowest } \\
\text { fitness score) is eliminated. } \\
\text { - The mutation operator is applied with some probability. } \\
\text { - All phases are repeated until an acceptable solution } \\
\text { evolved. }\end{array}$ \\
\hline Main results & $\begin{array}{l}\text { - A possible solution evolved after } 165 \text { generations. The } \\
\text { duration of all therapies was } 335 \text { minutes. } \\
\text { - Idle time for } 3 \text { devices is } 10,5 \text {, and } 30 \text { minutes, respec- } \\
\text { tively, without affecting the total time. } \\
\text { - The maximum overall waiting time is } 15 \text { minutes for } \\
\text { one patient. } \\
\text { - The overall average waiting time is } 5 \text { minutes. }\end{array}$ \\
\hline
\end{tabular}

Table 2 summarizes the description of evidence for the three articles that reported techniques used to improve nurse scheduling (Group 2). These three studies measured the reduction in time necessary to develop the schedule:

1. The studies used three different methods for staff scheduling: an auctionoptimization method, a mathematical model, and an indirect genetic algorithm.

2. The outcomes showed reduced time allocated to staff scheduling.

3. All studies used different constraints such as the number of hours worked in a week and the number of nights worked in a week.

4. All studies were performed in the USA, Canada, and UK.

Additionally, the descriptions of all the models in studies are summarized in Table 2. 
Table 2. Description of evidence for nurse scheduling (Group 2)

\begin{tabular}{|c|c|}
\hline Source & Description \\
\hline \multicolumn{2}{|c|}{ Beaulieu et al., 2000 [40] } \\
\hline Intervention & $\begin{array}{l}\text { Use of a mathematical model to facilitate preparing a schedule for } \\
\text { physicians in emergency room. }\end{array}$ \\
\hline Outcome & $\begin{array}{l}\text { Reduction in time and effort required to develop a six-month } \\
\text { schedule. }\end{array}$ \\
\hline Study population & 20 physicians, including 15 working full-time. \\
\hline Methods & $\begin{array}{l}\text { The model is formulated as a single-objective optimization model, } \\
\text { which seeks to minimize a weighted sum of all deviations. } \\
\text { - Four groups of constraints are defined: } \\
\circ \text { Compulsory constraints (e.g., one physician must be } \\
\text { assigned to one shift at the time); } \\
\circ \text { Ergonomic constraints (e.g., limits on number of weekly } \\
\text { hours of certain types of shifts); } \\
\circ \text { Distribution constraints (e.g., seniority), and } \\
\circ \text { Goal constraints (e.g., number of worked hours per week). } \\
\text { Two programs are developed: } \\
\circ \text { One to generate the model in a format accessible to the } \\
\text { branch-and-bound software, by reading input file; } \\
\circ \text { The other one to read the solution, create an output file and } \\
\text { to identify violations of the ergonomic rules. }\end{array}$ \\
\hline Main results & $\begin{array}{l}\text { - This approach can take into account more rules than any human } \\
\text { expert. } \\
\text { - It violated ergonomic rules } 40 \% \text { less than the human expert } \\
\text { ( } 111 \text { compared with } 185 \text { violations by human expert). } \\
\text { - It is faster than a manual method. }\end{array}$ \\
\hline
\end{tabular}

Aickelin and Dowsland, 2004 [41]

Intervention

Outcome

Study population

Methods
- Use of an indirect genetic algorithm method and a heuristic method in nurse scheduling.

- Minimize total preference cost of all nurses

- 52 real hospital datasets

- The problem is formulated as an integer linear programming.

- The objective function is:

$$
\operatorname{MIN} \sum_{i=1}^{n} \sum_{j \in F(i)}^{m} p_{i j} x_{i j}
$$

where $x_{i j}$ is decision variables, and equals " 1 " if nurse $i$ works shift pattern $j$, and " 0 " otherwise; $p_{i j}$ is preference nurse; $n$ is number of nurses; $m$ is number of shift patterns. 
Table 2. Description of evidence for nurse scheduling (Group 2) (Contined)

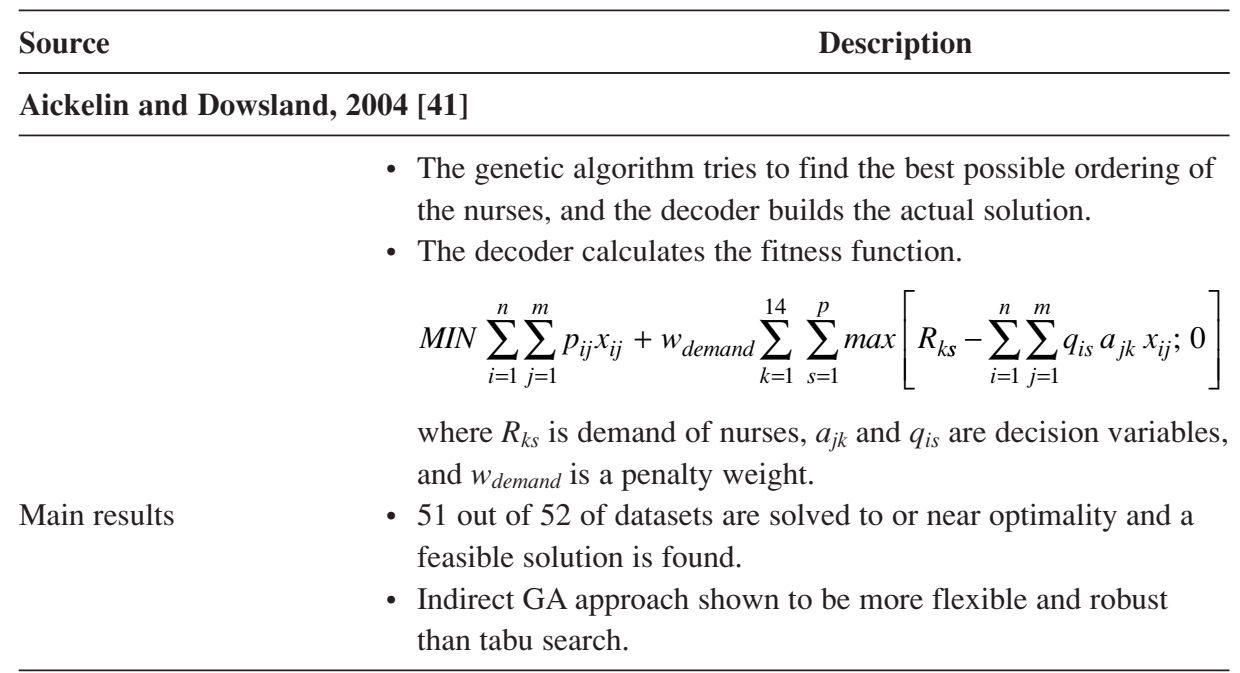

DeGrano et al. 2009 [42]

Intervention

Outcome

Study population

Methods

Main results
Use of auction and optimization model in nurse scheduling to accommodate individual preferences.

Minimize the time allocated to nurse scheduling.

28 full-time nurses with 36 h/week, 6 full-time with 40 h/week, six part-time working 20-28 h/week, 28 nurses working occasionally, 5 traveler nurses working $36 \mathrm{~h} /$ week.

- A weight is assigned for each shift (e.g., 1 for a 4-h shift, 2 for 8-h shift, 3 for a 12-h shift, and 6 for day-off). These weights are converted to bid points.

- Each nurse submits a preferred scheduled. Points are assigned to the schedule. Maximum number of points is 400 .

- Each nurse submits a bid.

- Two optimization models are used after the bid:

For the award step, the objective function maximizes the point value of bids awarded to the candidate winners.

- For schedule completion, the objective function awards bids which were not selected as candidate winners but can create a feasible assignment.

- It took $2.073 \mathrm{~s}$ to determine the candidate winner and generate the formulation. For award stage, it took 2 minutes and $55 \mathrm{~s}$. To complete the schedule, it took $5.74 \mathrm{~s}$.

- Overall it took about 3 minutes to generate the schedule, which is much faster than the manual method.

- The auction-optimization approach can account for both, the nurse preferences and the hospital constraints, and generate a good schedule.

- It fulfilled $98.27 \%$ of "on" requests and $95.51 \%$ of "off" requests. 


\subsection{Critical Appraisal}

Studies were evaluated based on scheduling improvement through minimizing patient waiting time, appointment span, and idle time for doctors. The overall critical appraisal referred to in Figures 2 and 3 applies to the overall quality of a study. For Group 1, the first six studies, Figure 2 shows that the studies ranged from a low $(0.24$ out of a maximum of 2.0 for the study by Kaandorp \& Koole [36]) to an average (0.97 for the study by Ogulata et al. [38]). For Group 2, Figure 3 shows that all three studies are with marginal quality (between 0.79 and 0.86 out of a maximum of 2.0).

The study quality, as measured by critical appraisal, was divided into reporting, subject selection, measurement quality, data analysis, and generalization of the study. The details of critical appraisal of the studies in Group 1 are shown in Figure 4. The following information was deduced:

1. All studies somewhat described the reporting elements, with the exception of that by Kaandorp and Koole [36] with a score of 0.38, which did not report any use of real data or confounders/covariates.

2. Most studies gave inadequate details about data selection, with the general score ranging from 0 to 1.00 . Only the study by Ogulata et al. [38] described the use of patient's data.

3. All studies were average in terms of measurement quality (ranging between 0.86 and 1.14 from a maximum of 2), with the exception of Isken and Rajagopalan [35] and Chakraborty et al. [39] which did not report any measurements.

4. The scores for data analysis methods were quite low, being below average or zero (not reported).

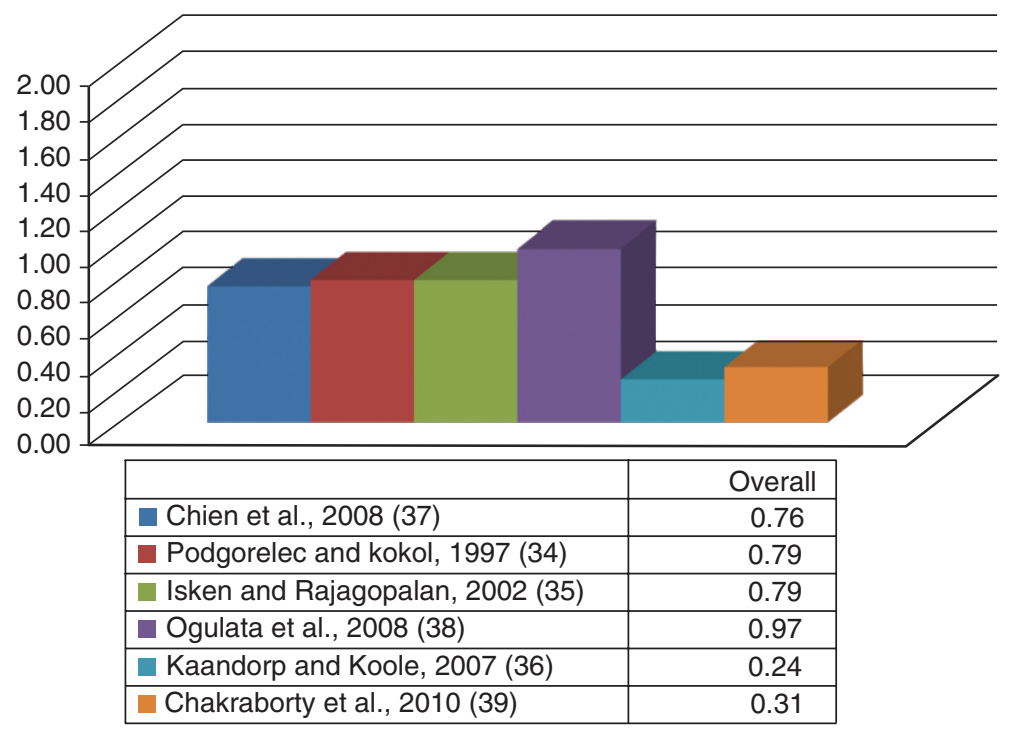

Figure 2. Overall critical appraisal scores for patient scheduling studies in Group 1 . 
5. The scores of study generalization were 2 for all except the study by Chakraborty et al. [39] and Isken and Rajagopalan [35] that had a score of zero. This was due to the unreported participation rate. A score of 2 means that the results of the study may be applicable to the eligible population or other relevant groups.

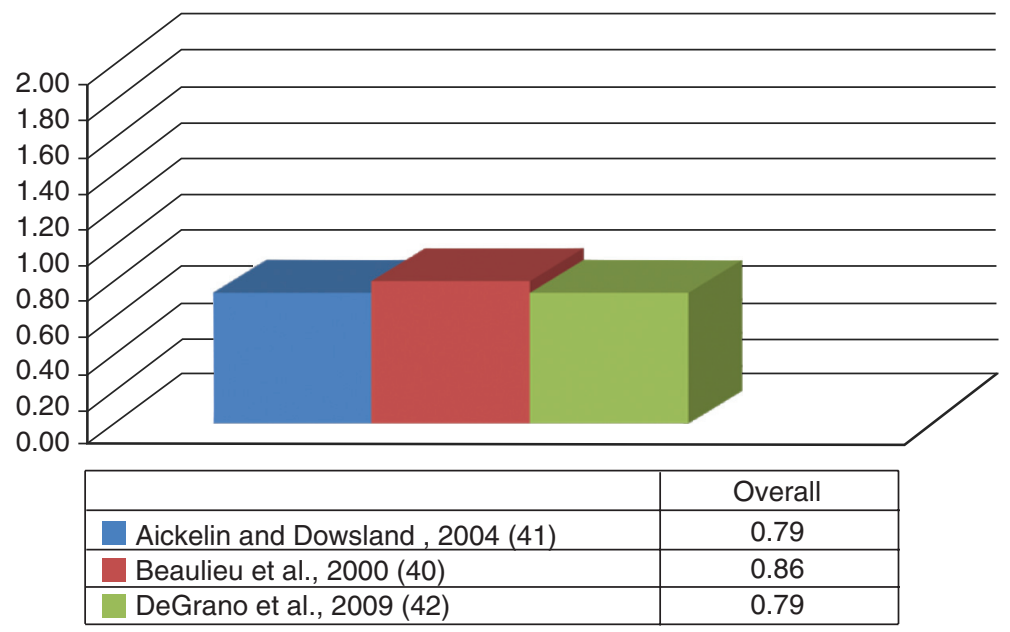

Figure 3. Overall critical appraisal scores for nurse scheduling studies in Group 2.

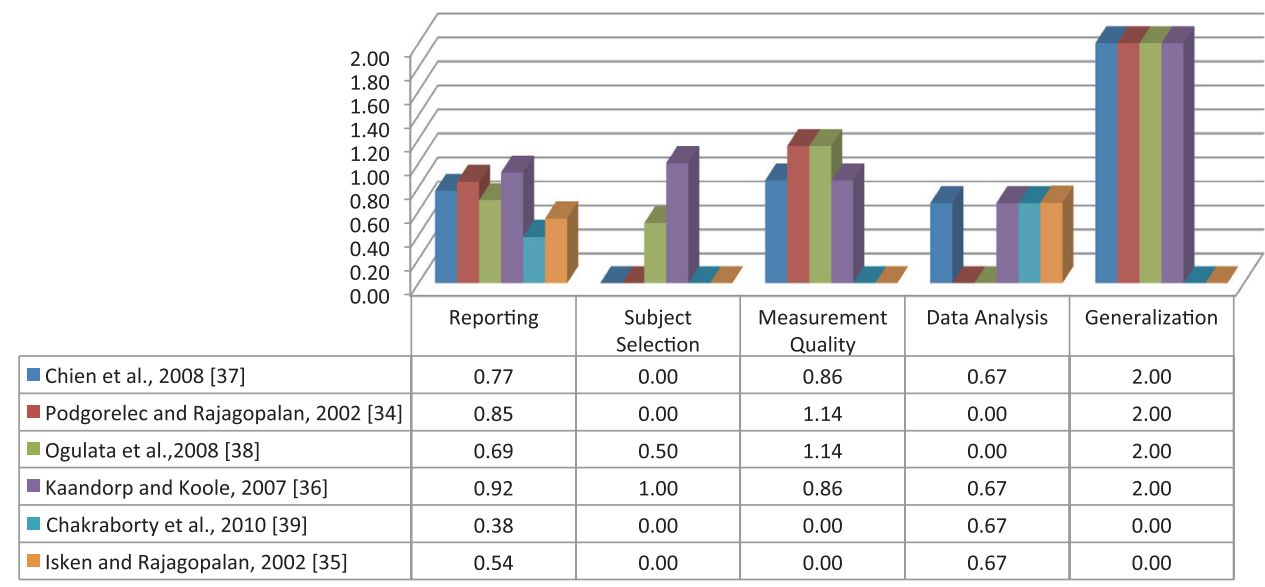

Figure 4. Study quality scores for patient scheduling studies in Group 1. 


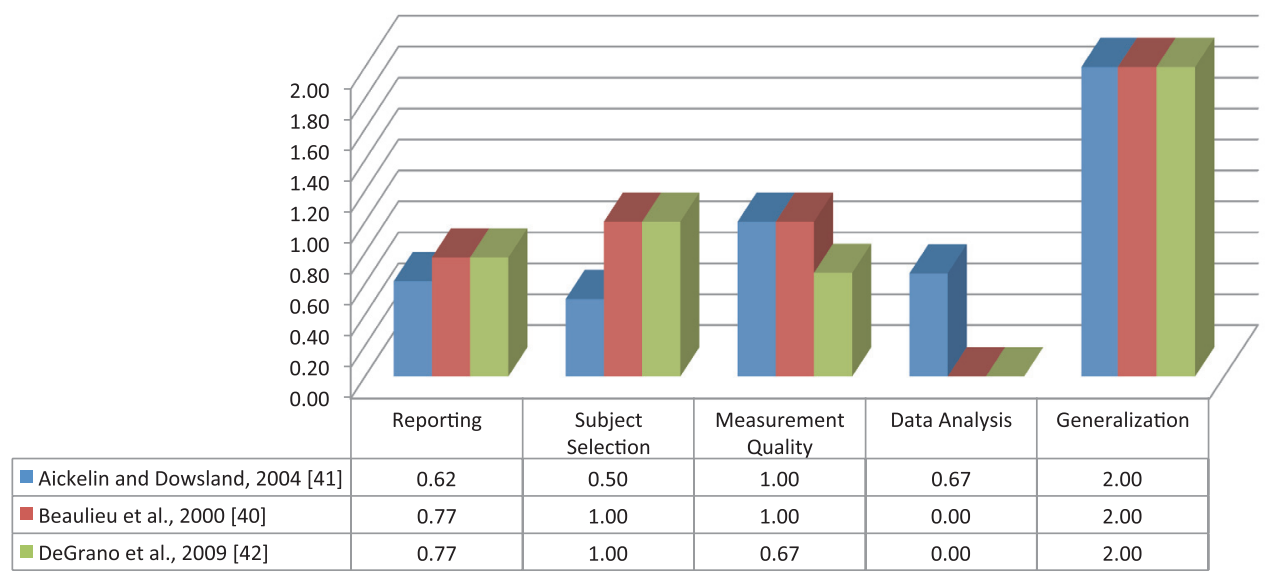

Figure 5. Study quality scores for nurse scheduling studies in Group 2.

The details of critical appraisal of the studies in Group 2 are shown in Figure 5.

The following conclusions were deduced:

1. The scores of reporting elements were below average (0.62-0.77).

2. The subject selection scores ranged from marginal $(0.5$ for Aickelin and Dowsland [41] to average for Beaulieu et al. [40] and DeGrano et al. [42].

3. The measurement quality scores were marginal to average for all studies, between 0.67 and 1.00 .

4. The scores for data analysis were low to marginal (0 to 0.67 ).

5. The scores of study generalization are maximum for each, as all participation rates were reported as being $100 \%$.

The measurement quality was divided into quality exposure, blind measurement, outcome, and observation period. Because the studies were only intervention studies, the answers to the questions dealing with exposure were not applicable, resulting in scores that were not reported for this part. The outcome scores were, on average, marginal (0.67). The observation period had a score of two for all studies, except Kaandorp and Koole [36] (score of 0).

\subsection{Description of Models}

\subsubsection{Mathematical Modelling and Optimization}

Mathematical modelling is based on the optimization concepts of linear programming. The objective of mathematical modelling is to minimize or maximize the objective function. It has to take into account a number of constraints defined by the problem. In general, optimization techniques include linear programming, integer programming (a 
linear programming technique that requires the variables to be integers), mixed integer programming, dynamic programming, constructive algorithms and more.

In this review, four studies that fall into this category were two studies that deal with physician and nurse scheduling [40, 42] and two studies that deal with patient/staff scheduling [38, 39]. Per optimization methodology, all studies defined the variables that were the inputs to the model, and the objective functions.

Beaulieu et al. [40] defined a multi-objective integer model that had multiple objectives and all the solutions had to be integers. The objective function sought to minimize a weighted sum of all deviations.

Ogulata et al. [38] used three hierarchical mathematical models with the objective of maximization of the number of patients seen in one week, obtaining a balanced distribution of patients among physicians, and minimization of patient waiting time. The mathematical models were applied in three different stages: weekly patient selection, assignment of physiotherapists, and patient scheduling.

The study by Chakraborty et al. [39] introduced sequential clinical scheduling, which can be formulated using dynamic programming. This study was based on an earlier work [43] which had maximization of the expected revenue for patients seen minus the cost for patient waiting and staff revenue as the objective function, including the no-show appointments in the scheduling model. This study was further adopted in subsequent research $[44,6]$ featuring the same objective function and constraints but different distribution of the numbers of patients' no-shows, whereas Chakraborty et al. study [39] considered that the no-show patients' distribution is homogeneous.

DeGrano et al. [42] took a different approach in his optimization model. In the initial stage of the model, there was an auction where nurses could bid the shifts they desired. In the second stage, the model awarded the shifts and did the scheduling. The objective for the award model was to maximize the point value of bids awarded to the candidate winners. The objective for the assignment model was to seek award bids which were not selected as candidate winner but can create a feasible assignment.

\subsubsection{Genetic Algorithms Used in Patient and Nurse/Physician Scheduling}

Genetic algorithms (GAs) are heuristic search methods used in solving complex search and optimization problems. GA's are based on the mechanism of natural selection and genetics. Often, they are able to find the optimal solution in more complex search spaces (the set of possible solutions) and can present significant benefits over other search and optimization techniques [34]. Three studies used genetic algorithms for scheduling patients or nurses: Podgorelec and Kokol [34]; Chien et al. [37]; and Aickelin and Dowsland [41]. Genetic algorithms typically involve six steps: 1) selection of initial population, 2) reproduction, 3) crossover, 4) mutation, 5) evaluation, and 6) selection of solution. All of the three studies used the same GA described below:

- The initial population can be selected randomly or through a seeded population. Podgorelec and Kokol [34] used a seeded population by filling the table with therapies in a random time order. Chien et al. [37] used a local search heuristic to derive the sequence of patients and therapies that represented the chromosome. 
Aickelin and Dowsland [41] built the chromosome as permutations of individual nurses.

- The reproduction step is explained in only two articles. Podgorelec and Kokol [34] assigned negative points to each individual selected from the initial population based on the values of some parameters. The fewer the negative points an individual had, the better chances the individual had to be selected for crossover. In Chien et al. [37], chromosomes were randomly selected.

- The crossover step was described in Podgorelec and Kokol [34] and Chien et al. [37]. The first study used the method of cutting chromosomes into different parts along the timeline and then randomly putting them together. The second study used a modified version of preserving an order-based crossover. After individuals were selected from the initial population, all precedence-dependent therapies were identified. These therapies were copied into the offspring chromosome at the same position. The remaining therapies were copied from parent 2 into the offspring following the same order.

- The mutation step was described in Podgorelec and Kokol [34] as a mutation between two random activities. In Chien et al. [37], the mutation procedure was performed by randomly selecting a therapy from a parent chromosome. The leftmost and rightmost positions were searched to determine an interval within which the selected therapy could mutate without violating the precedence constraint. The therapy was then inserted into the selected position.

- The evaluation method was different in each study, but all used a fitness score to evaluate the chromosomes. Podgorelec and Kokol [34] and Chien et al. [37] used a combination of maximum waiting time and the makespan (the total duration of all services for a day) as the evaluation function. Aickelin and Dowsland [41] used the total preference cost of all nurses as an evaluation function.

- The selection of a solution was made based on the evaluation function. In the two studies involving patient scheduling [40, 42], the solution with the minimum waiting time and the lowest makespan was chosen. In the studies involving nurse scheduling $[38,39]$, the solution with the minimum total preference cost was chosen.

\subsubsection{Local Search and Data Mining Models}

Two articles dealt with the use of local searches and data mining techniques for patient and nurse scheduling: Kaandorp and Koole [36] and Isken and Rajagopalan [35]. The local search method used by Kaandorp and Koole [36] started with a feasible solution and searched for a better solution in its neighbourhood until a local minimum was found. The solution found was not a global minimum, but with a well-chosen neighbourhood, it was possible to find the global minimum. To find a feasible solution, the authors used a mathematical model type of scheduling that calculated patient mean waiting time, physician idle time, and tardiness. The objective was to minimize the sum of the three variables. The number of solutions given by this model was so large that a search algorithm was needed to select the best one. 
Isken and Rajagopalan [35] used a data mining technique, specifically clustering, such as K-means. The authors started with the concept that patients have different individual needs such as different types of treatments or sequences of treatments, and consequently, different resources need to be allocated to individual patients. This would be too big of a problem to solve, so the solution would be patient classification in groups that have the same needs.

To classify patients in groups, Isken and Rajagopalan [35] used the number of total hours spent in different categories of hospital units, and the path and associated lengths of stay as input variables. The authors also used Diagnostic Related Groups (DRG's) and Clinical Classification Software (CCS) that provided information about the diagnoses and procedures. The K-means clustering method was employed to classify patients. K-means is an applied clustering algorithm in unsupervised classification problems to find $k$ optimal clusters in a data set. The algorithm includes the following steps:

- Initialization - Choose the number of clusters, $k$.

- Find new clusters - Randomly generate $k$ clusters and determine the cluster centers, or directly generate $k$ random points as cluster centers.

- Assign each point to the nearest cluster center.

- Recompute the new cluster centers.

- Repeat the two previous steps until some convergence criterion is met.

\section{DISCUSSION}

A key point in improving healthcare delivery is the improvement of process performance. Part of process performance is balancing the demand and resources. In the healthcare system, the demand is the number of patients to be seen every day and the resources are the nurses, physicians, rooms, and instrumentation that are available. Balancing demand and resources will direct us towards the problem of scheduling patients and resources. In this paper, we focused exclusively on methods for patient and staff scheduling.

All of the computerized methods developed in the recent decades try not only to improve the quality of service, but also to help schedulers do their work faster [37]. The attention of the new methods is focused not only at staff scheduling, but equally at patient scheduling. These methods aim at the reduction of patient waiting time (indirect and direct) and total time spent in the medical facility.

As Podgorelec and Kokol [34] noted, regardless of the methods used, there are basic rules that must be followed for successful development of a qualitative and effective automated scheduling system, such as feasibility of all obtained solutions, and fulfillment of all constraints. Additionally, an adequate solution must be found in a reasonable amount of time. An indispensable property of the scheduling technique must be the capability of solving general and independent problems, i.e., the applicability to various situations, especially in an unpredicted situation. For example, it should allow the system to search for solutions even when activities already scheduled are cancelled. 
The articles included in this review presented different methods for patient and nurse/physician scheduling: mathematical modeling/optimization, genetic algorithm, local search, and data mining. All these methods had common objectives:

- $\quad$ To decrease patient waiting time [34, 35, 36, and 37]

- $\quad$ To reduce physician idle time [36]

- To increase equipment utilization $[23,37]$

- $\quad$ To process the maximum number of patients [38]

- To distribute patients equitably among physicians [38]

- $\quad$ To reduce the time and effort in schedule construction [40, 41]

Achieving these objectives will result, indirectly, in an improvement of healthcare quality and a reduction in cost, parameters that are essential to the wellbeing of healthcare system. These models take into account a number of variables such as the number of open slots per day, number of days in the schedule, length of each appointment, number of patients to be scheduled each day, number of patients assigned to each staff member, overall duration of activities, process starting time and completion time, earliest starting time and latest completion time, number of shifts available during the schedule period, and resource utilization cost.

The studies included in this review proved that data mining approaches were more successful in achieving the objectives of scheduling than standard (manual) scheduling methods. Besides achieving the objectives listed above, data mining approaches showed new advantages that were not directly related to the main objectives. For example, in the study by DeGrano et al. [42], the auction-optimization approach accommodated the preferences of individual nurses. Furthermore, the schedule could be generated in a reasonable amount of time. In the study by Isken and Rajagopalan [35], the k-means performed well completing the task in 3-5 seconds. Also, it showed that data preparation is an important step in scheduling and has great implications in the quality of the solution. Some approaches such as the study by Aickelin [40], show that the use of genetic algorithms can be more flexible in implementation than Tabu Search.

In addition to the variables used in these models, variables that are related to patient behavior should be used as inputs in a model. All the models included in this review considered that patients and staffs were always on time; however, this is not true in real life. Occasionally, physicians are late for their clinics [24] or patients are late for their appointments. Many of the missed appointments involve patients not showing up without canceling in advance. Reviews in the scientific literature concluded that the noshow rates may be around 20\% [47] and vary between 15-30\% in general adult and pediatric clinics [48], and 2-15\% in private practices [46]. Missed patient appointment (no-show) has an adverse effect on resource utilization in healthcare services, resulting in under-utilization of clinic capacity [49], loss in revenue, inefficient scheduling, and underutilization of personnel [51]. While previous efforts have identified some relevant elements of the systems, they fail to provide a holistic, quantitative approach combining the organization scheduling system and patient behaviour into a common framework. 
All the models included here have accounted for a series of constraints. Beaulieu et al. (40) grouped the constraints into four distinct categories. These types of constraints were found in the other nurse scheduling studies [40,42]. The four constraint categories were as follows:

1. Compulsory constraints: (a) one physician/nurse must be assigned to each shift period; (b) a physician/nurse cannot work more than a shift per day; and c) a physician/nurse assigned to a night shift cannot be assigned to the following day shift.

2. Ergonomic constraints: (a) upper limits on the number of weekly hours of certain types of shifts; (b) limits on the number of successive working days; c) the need for leave on the Monday after working the weekend; d) the need for three consecutive days of leave if physician/nurse works for three consecutive night shifts.

3. Distribution constraints: employee seniority.

4. Goal constraints: (a) a physician/nurse should work a specified number of hours per week; (b) certain types of shifts must be fairly distributed among physicians/nurses.

The patient scheduling studies also had constraints as follows:

- No patient can perform more than one activity at a time.

- Each resource (personnel or device/instrument) can be used by only one patient at a time.

- Each activity has to be performed in only one continuous time interval.

- Every activity can be performed only with a specific resource.

- Some activities have to be performed in an exact time order.

- Makespan must not exceed the service time of the healthcare facility.

Incorporation of all these constraints helps find the most feasible solution, but does not guarantee the best solution to use. More criteria can be included in the evaluation function to improve the selection of the fittest solution. These constraints can be related to patient characteristics or behaviour.

The most important advantage of using these new methods in scheduling of healthcare is that it takes much less time than the traditional manual methods. Also, adjusting the importance of each input that may affect the quality of the final solution makes these methods more likely to be included in scheduling tools. They can solve much more complex problems than the traditional methods by incorporating more input variables and more constraints. These methods can account for all constraints simultaneously while traditional methods can account for only one constraint at a time.

There are some limitations in using these new methods. All artificial intelligence (AI) techniques develop rules based on the input and constraints. Since these inputs and 
constraints are specific to each healthcare facility, one may ask the question, "Can these AI models be applied to a wide range of healthcare facilities?" The challenge remains in developing procedures that can resolve the conflict arising from using different sets of rules developed by multiple experts [55].

Other considerations that have to be taken into account are related to patients such as indirect waiting time, late cancellation and no-show, emergency walk-ins and patient preferences [3]. The scheduling system can affect both direct waiting time and indirect waiting time. Both direct and indirect times may pose a serious risk for the patient safety. Both no-shows and walk-ins influence the scheduling system. No-shows create gaps in the system that reduce revenue without reducing cost. Also, by not showing for an appointment, a patient denies an appointment to another patient and increases the indirect waiting time. Walk-ins occur randomly and may increase waiting time for patients and overload nurses/physicians. However, managing no-shows and walk-ins may be a challenging task. Finally, none of the reviewed models take patient preferences into account in scheduling. Although preferences differ from one person to another, one model [35] created groups of patients (clusters) with similar preferences and then used these preference groups in developing a schedule.

\section{CONCLUSION}

The methods covered in this literature review represent new approaches to solving the problem of scheduling in healthcare. They were adopted from other areas, such as manufacturing or transportation. Due to the complex and dynamic healthcare environment, their implementation has to be carefully planned. The use of data mining models was proven to be successful in improvement of healthcare scheduling. The advantage of data mining techniques is improved competitiveness of the healthcare scheduling system by discovering patterns and trends in large data sets generated by healthcare systems, and using these patterns and trends in decision making. Data mining is considered to be the best approach so far for dealing with the complex healthcare scheduling issues.

One limitation of these scheduling methods was that they were designed for specific providers, thus lacking generality. Another limitation was that each method concentrated on a single measure of performance at a time, such as time-based measures (waiting time, idle time). More external variables, such as patient behaviour, should be taken into account in developing improved scheduling methods.

One of the problems yet to be solved is inclusion of no-show, cancelation, walk-ins in a scheduling system. Accurately predicting no-shows, cancelations, and walk-ins could significantly improve scheduling performance, and consequently increasing provider revenue. Future research should forecast no-show, cancelation, and walk-ins and suggest techniques to be incorporated into the scheduling system.

The use of data mining techniques to assist scheduling in healthcare can have a great impact on the scheduling system. This impact can be attributed to: (a) the large number of factors that can be taken into account; (b) weighting the influence of each factor; (c) quantifying and processing the large number of variables; and (d) reducing the time necessary for scheduling. 


\section{CONFLICT OF INTEREST}

The authors indicated no potential conflict of interest.

\section{REFERENCES}

[1] Bureau of Labor Statistics 2009. Estimated quarterly revenue for employer firms, seasonally adjusted. www.census.gov/qss. Accessed September 2009.

[2] Plunkett Research Ltd. Industry statistics, trends and in-depth analysis of top companies. http://www.plunkettresearch.com/Industries/HealthCare/HealthCareTrends/tabid/294/Default.aspx. Accessed September 2009.

[3] Gupta, A., and Denton, B. Appointment scheduling in health care: challenges and opportunities. IIE Transactions. 2008, 40, 800-819.

[4] Sweeney, D.R. Your office: a lot of things will have to change. Medical Economics. 1996, 73(7), 97-102.

[5] Wright, P.D., Bretthauer, K.M., and Coté, M.J. Reexamining the nurse scheduling problems: staffing ratios and nursing shortages. Decision Science. 2006, 37, (1), 39-70.

[6] Turkan, A., Zeng, B., Muthuraman, K., and Lawley, M. Sequential clinical scheduling with service criteria. European journal of Operational Research. 2011, 214, 780-795.

[7] Fei, H., Meskens, N., and Chu, C. A planning and scheduling problem for an operating theatre using an open scheduling strategy. Computers and Industrial Engineering. 2010, 58, 221-230.

[8] Ceschia, S., and Schaerf. A. Local search and lower bounds for the patient admission scheduling problem. Computers and Operations Research. 2011, 38, 1452-1463.

[9] Huang, X. Patients Attitude towards waiting in an outpatient clinic and its applications. Health Services Management Research. 1994, 7, 2-8.

[10] Jackson, A.R. A waiting time survey in general practice. Australian Family Physician. 1991, 20(12), 1744-1750.

[11] Wojtys M., Edward, L. S., Overgaard, K. A., Agbabian, J. Applying lean techniques to improve the patient scheduling process. Journal for Healthcare Quality. 2009, 31, 10-16.

[12] Qu, X., and Shi, J. Modeling the effect of patient choice on the performance of open access scheduling. International Journal of Production Economics. 2011, 129, 314-327.

[13] Murray, M., and Berwick, D.M. Advanced access: reducing waiting and delays in primary care. Journal of American Medical Association 2003. 289, 1035-1040.

[14] Kachhal, S. K. Industrial engineering Applications in Health Care Systems. In Handbook of Industrial Engineering. Technology and Operations Management. $3^{\text {rd }}$ Edition. John Wiley and Sons, Inc. New York, 2001.

[15] Lindley, D.V. The theory of queues with a single server. Proceedings Cambridge Philosophy Society. 1952, 48, 277-289.

[16] Bailey, N.T.J. A study of queues and appointment systems in hospital out-patient departments, with special reference to waiting-time. Journal of Royal Statistical Society. 1952, A 14, 185-199.

[17] Jansson, B. Choosing a good appointment system: a study of queues of type (D/M/1). Operations Research. 1966, 14, 292-312.

[18] Soriano, A. Comparison of two scheduling systems, Operations Research, 1966, 14, 388-397.

[19] Mercer, A. A queuing problem in which arrival times of the customers are scheduled. Journal of the Royal Statistical Society Series B. 1960, 22, 108-113.

[20] Fries, B. and Marathe, V. Determination of optimal variable-sized multiple-block appointment systems. Operations Research. 1981, 29(2), 324-345.

[21] Brahimi, M. and Worthington, D. J. The finite capacity multi-Server queue with inhomogeneous arrival rate and discrete service time distribution and its application to continuous service time problems. European Journal of Operational Research. 1991, 50(30), 310-324. 
[22] Liu, L. and Liu, X. Block appointment systems for outpatient clinics with multiple doctors. Journal of Operational Research Society. 1998, 49(12), 1254-1259.

[23] Denton, B. and Gupta, D. A sequential bounding approach for optimal appointments scheduling. IIE Transactions. 2003, 35, 1003-1016.

[24] Welch, J.D., and Bailey, N.T.J. Appointment systems in hospital outpatient departments. Lancet. 1952, 259, 1105-1109.

[25] Welch, J.D. Appointment systems in hospital outpatient departments. Operational Research Quarterly. 1954, 15(3), 224-232.

[26] Fetter, R. and Thompson, J. Patients' waiting time and doctors' idle time in the outpatient setting. Health Services Research. 1966, 1, 66-90.

[27] Vissers, and Wijngaard, J. The outpatient appointment system: design of a simulation study. European Journal of Operational Research. 1979, 3(6), 459-463.

[28] Ho, C. and Lau, H. Minimizing total cost in scheduling outpatient appointments. Management Science. 1992, 38(12), 1750-1764.

[29] Ho, C., Lau, H, and Li, J. Introducing variable-interval appointment scheduling in service systems. International Journal of Production \& Operations Management. 1995, 15(6), 59-69.

[30] Ho, C. and Lau, H. Evaluating the impact of operating conditions on the performance of appointment scheduling rules in service systems. European Journal of Operational Research. 1999, 112(3), 542-553.

[31] Klassen, K. J. and Rohleder, T.R. Scheduling outpatient appointments in a dynamic environment. Journal of Operations Management. 1996, 14(2), 83-101.

[32] Rohleder, T. R. and Klassen, K.J. Using client-variance information to improve dynamic appointment scheduling performance. Omega. 2000, 28(3), 293-302.

[33] Genaidy, A.M. Cancer risk among firefighters: Epidemiologic evidence. $\mathrm{PhD}$ dissertation, University of Cincinnati, 2010.

[34] Podgorelec, V., and Kokol, P. Genetic algorithm based system for patient scheduling in highly constrained situations. Journal of Medical Systems. 1997, 21(6), 417-427.

[35] Isken, M.W., and Rajagopalan, B. Data mining to support simulation modeling of patient flow in hospitals. Journal of Medical Systems. 2002, 26(2), 179-197.

[36] Kaandorp, G.C. and Koole, G. Optimal outpatient appointment scheduling. Health Care Management Science. 2007, 10, 217-229.

[37] Chien, C-F, Tseng, F-T, and Chen, C-H. An evolutionary approach to rehabilitation patient scheduling: a case study. European Journal of Operational Research. 2008, 189, 1234-1253.

[38] Ogulata, S.N., Koyuncu, M., and Karakas, E. Personnel and patient scheduling in the high demand hospital services: a case study in the physiotherapy service. Journal of Medical Systems. 2008, 32, 221-228.

[39] Chakraborty, S., Muthuraman, K., and Lawley, M. Sequential clinical scheduling with patient noshows and general service time distributions. IIE Transactions. 2010, 42, 354-366.

[40] Beaulieu, H., Ferland, J.A., Gendron, B., and Michelon, P. A mathematical programming approach for scheduling physicians in the emergency room. Health Care Management Science. 2000, 3, 193-2000.

[41] Aickelin, U., and Dowsland, K.A. An indirect genetic algorithm for a nurse scheduling problem. Computers and Operations Research. 2004, 31(5), 761-778.

[42] DeGrano, M. L., Medeiros, D.J., and Eitel, D. Accommodating individual preferences in nurse scheduling via auction and optimization. Health Care Management Science. 2009, 12, 228-242.

[43] Muthuraman, K. and Lawley, M. A stochastic overbooking model for outpatient clinical scheduling with no-shows. IIE Transactions. 2008, 40(9), 820-837.

[44] Zeng, B., Turkcan, A., Lin, J., and Lawley, M. Clinic scheduling models with overbooking for patients with heterogeneous no-show probabilities. Annals of Operations Research. 2010, 178, 121-144.

[45] Hall, R. Patient flow: the new queuing theory for healthcare. OR/MS Today. 2006, 33, 36-40. 
[46] Nolan, T., Schall, M., Berwick, D., and Roessner, J. Reducing delays and waiting times throughout healthcare system. Institute for Healthcare Improvement. Boston, MA, 1996.

[47] Bennett, K.J., and Baxley, E. The effect of a carve-out advanced access scheduling system on no-show rates. Family Medicine. 2009, 41(1), 51-56.

[48] Deyo, R. A. and Inui, T.S. Dropouts and broken appointments: a literature review and agenda for future research. Medical Care. 1980, 18, 1146-1157.

[49] Barron, W.M. Failed appointments: who misses them, why they are missed, and what can be done. Primary Care. 1980, 7, 563-574.

[50] Daggy, J., Lawley, M., Willis, D., Thayer, D., Suelzer, C., DeLaurentis, P-C., T, A., Chakraborty, S., and Sands, L. Using no-show modeling to improve clinic performance. Health Informatics Journal. 2010, 16, (4), 246-259.

[51] Macharia, W.M., Leon, G., Rowe, B.H., Stephenson, B.J., and Haynes, B. An overview of interventions to improve compliance with appointments keeping for medical services. Journal of the American Medical Association. 1992, 267, 1813-1817.

[52] Organization for Economic co-operation and development. http://www.oecd.org/home/0,3305, en 2649201185111111 l,00.html. Accessed 2011.

[53] U.S. Census Bureau. http://www.census.gov/. Accessed 2011.

[54] LaGanga, L. and Lawrence, S.R. Clinic overbooking to improve patient access and increase provider productivity. Decision Sciences. 2007, 38(2), 251-276.

[55] Sitompul, D., and Randhawa, S. U. Nurse scheduling models: a state-of-the-art review. Journal of Society for Health Systems. 1990, 2(1), 62-72. 



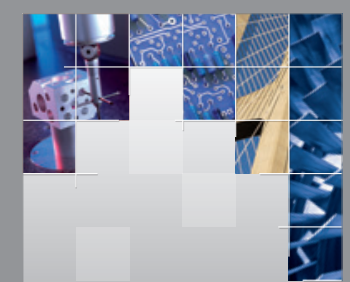

\section{Enfincering}
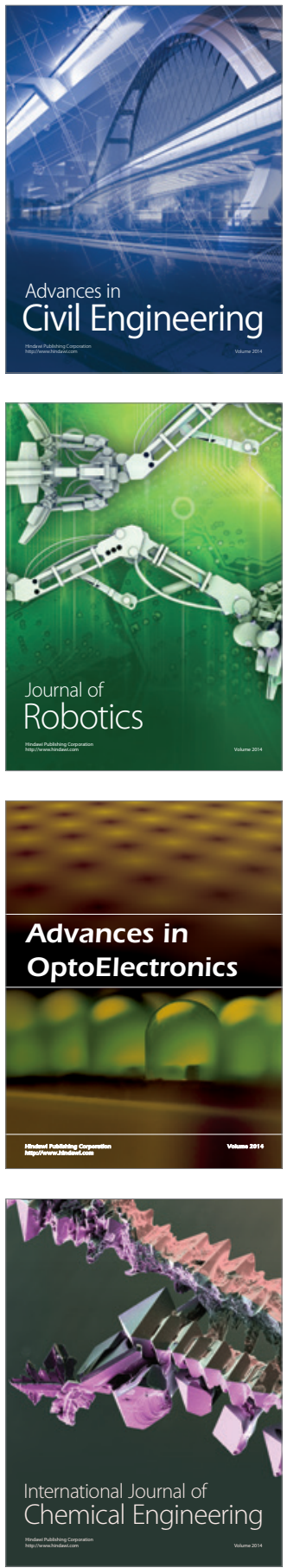

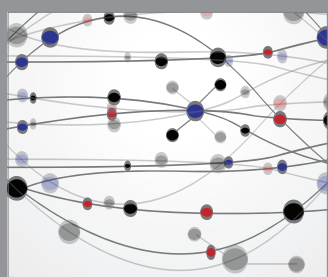

The Scientific World Journal

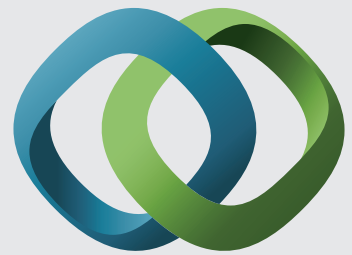

\section{Hindawi}

Submit your manuscripts at

http://www.hindawi.com
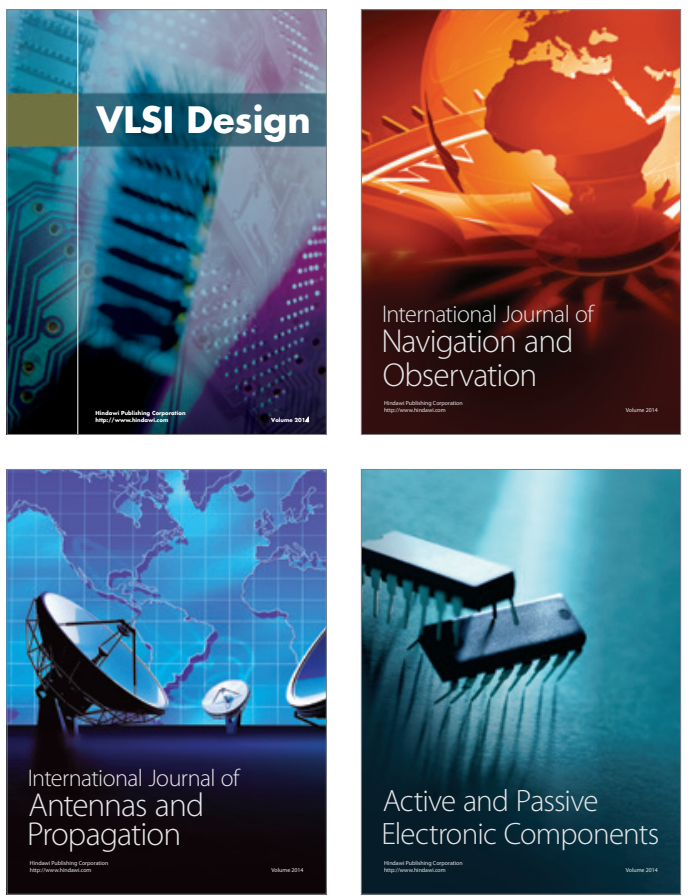
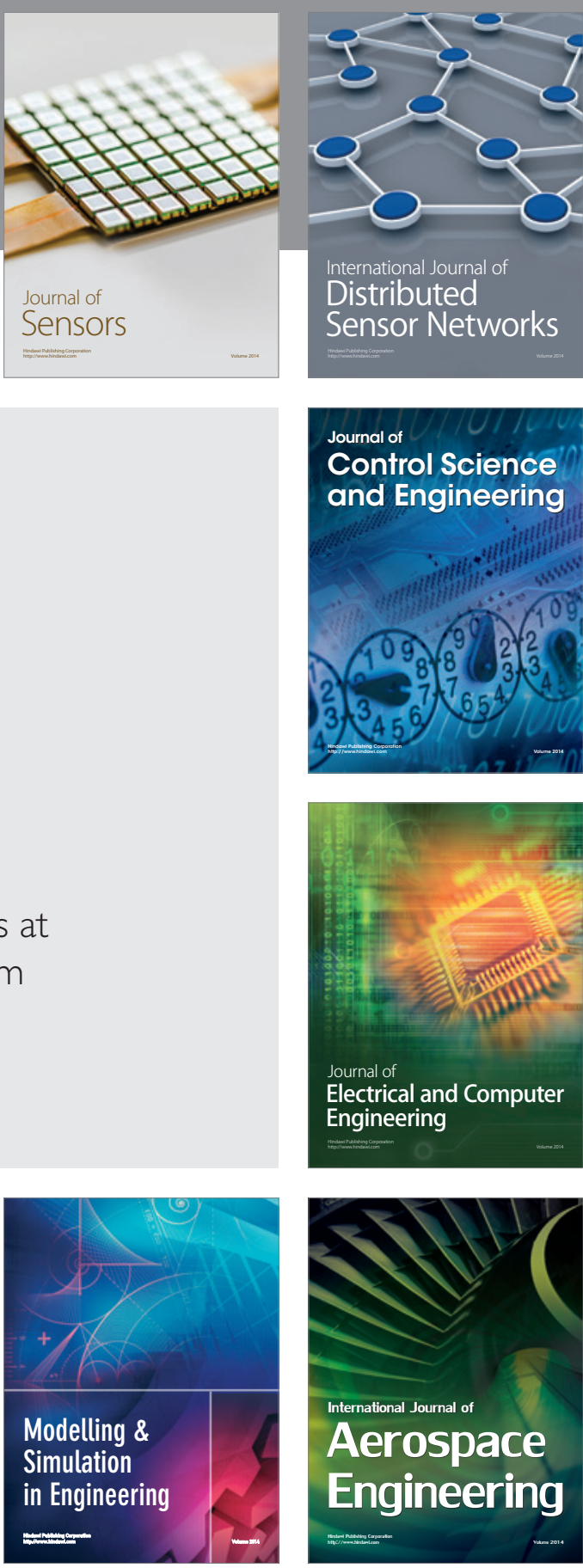

International Journal of

Distributed

Sensor Networks

Journal of

Control Science

and Engineering
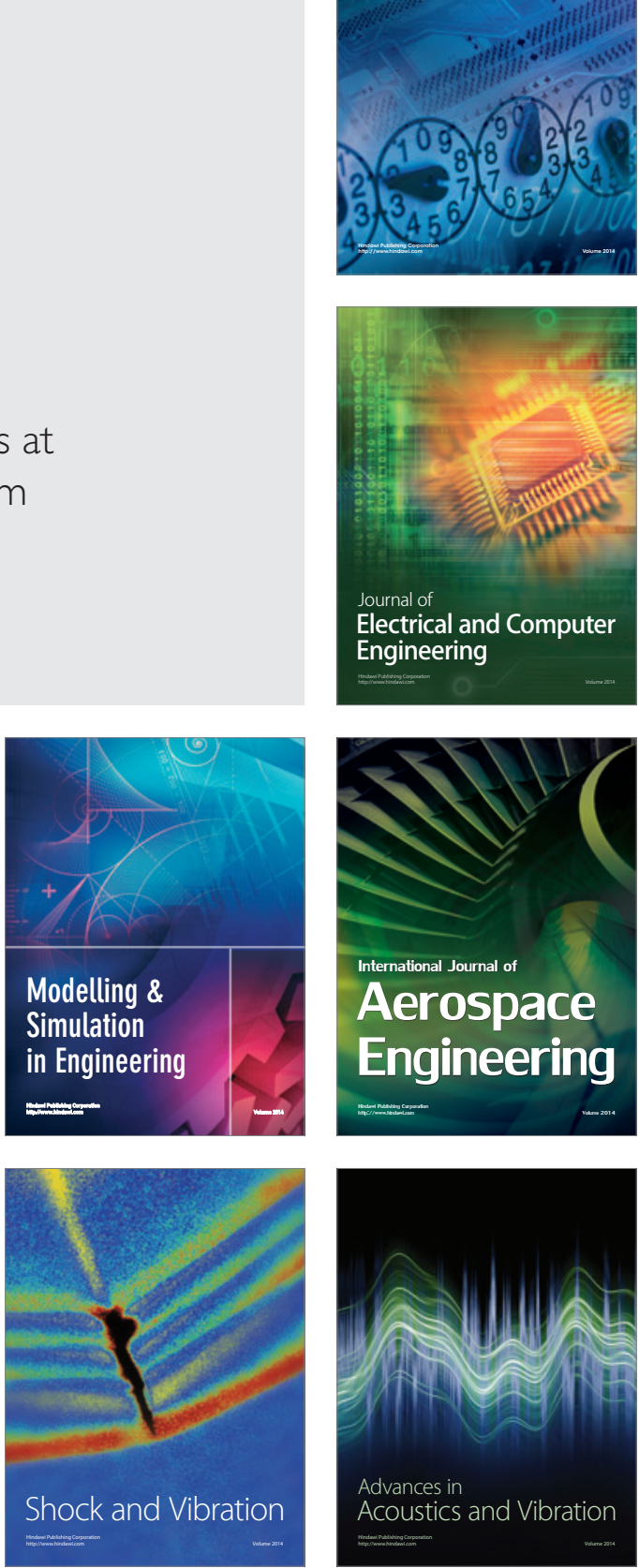